\title{
From Yang-Baxter Maps to Integrable Recurrences
}

B. Grammaticos, A. Ramani, C.-M. Viallet

To cite this article: B. Grammaticos, A. Ramani, C.-M. Viallet (2013) From Yang-Baxter Maps to Integrable Recurrences, Journal of Nonlinear Mathematical Physics 20:2, 260270, DOI: https://doi.org/10.1080/14029251.2013.813105

To link to this article: https://doi.org/10.1080/14029251.2013.813105

Published online: 04 January 2021 


\title{
From Yang-Baxter Maps to Integrable Recurrences
}

\author{
B. Grammaticos, A. Ramani, and C.-M. Viallet \\ IMNC, Université Paris VII \& XI, CNRS, UMR 8165, Bât. 440, 91406 Orsay, France \\ Centre de Physique Théorique, École Polytechnique, CNRS, 91128 Palaiseau, France \\ LPTHE, Université Pierre-et-Marie-Curie, CNRS UMR 7589, 4 place Jussieu, 75252 Paris cedex 05, France
}

Received 29 November 2012

Accepted 30 May 2013

\begin{abstract}
Starting from known solutions of the functional Yang-Baxter equations, we construct a series of nonautonomous integrable recurrences, "median graphs", and give their explicit solution.
\end{abstract}

PACS numbers: 02.30.Ik, 05.45.Yv

\section{Introduction}

A number of discrete integrable systems have been described in the literature in the last decades. They are mostly discrete versions of known integrable differential equations and partial differential equations, retaining fundamental features of integrability, as for example the existence of a sufficient number of invariant tori and hierarchies of associated integrable equations. Important classification results [1] have been obtained, both for the ordinary difference equations case (in particular integrable maps [2] and discrete Painlevé equations [3]) and for the partial difference equations (integrable lattice equations and Yang-Baxter maps among others [4-6]).

The present paper is motivated by recent results on Yang-Baxter maps obtained by Adler, Bobenko and Suris [5] and complemented by Papageorgiou, Suris, Tongas and Veselov [6]. Here we shall introduce a new dynamical interpretation of the Yang-Baxter maps, the "median graph reduction". Obviously, this is but one among many possible interpretations of Yang-Baxter maps. However, the one presented here has the interesting feature to lead to a set of linearisable mappings for which we are able to construct the explicit solution. This is made possible essentially thanks to a previous construction [7] of the explicit solution of the equation obtained here from a compatibility condition, equation which was initially derived in [8].

\section{The setting}

The underlying geometry behind the models is a two dimensional square lattice with variables associated to the edges. Each elementary cell is labelled by a pair of integers $(m, n)$, which is the pair of coordinates of the lower left corner of the cell. The elementary cell is depicted in Figure 1.

The variables $(x, y, u, v)$ are assigned to the bonds as shown in Figure 1. The models are defined by giving a $2 \rightarrow 2$ map $(x, y) \rightarrow(u, v)$, verifying the functional Yang-Baxter equations (aka YangBaxter maps). We start from the models described in $[4,6]$ and use the same labelling, namely the 


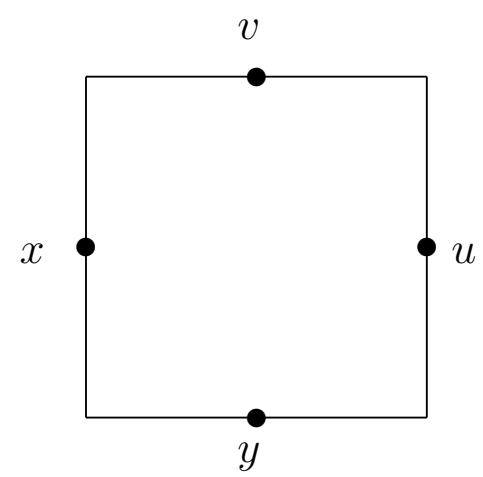

Fig. 1. Original cell

"F" and "H" families, which we reproduce here:

$$
\begin{gathered}
u=\alpha y P, \quad v=\beta x P \quad P=\frac{(1-\beta) x+\beta-\alpha+(\alpha-1) y}{\beta(1-\alpha) x+(\alpha-\beta) x y+\alpha(\beta-1) y} \\
u=\frac{y}{\alpha} P, \quad v=\frac{x}{\beta} P, \quad P=\frac{\alpha x+\beta-\alpha-\beta y}{x-y} \\
u=\frac{y}{\alpha} P, \quad v=\frac{x}{\beta} P, \quad P=\frac{\alpha x-\beta y}{x-y} \\
u=y P, \quad v=x P, \quad P=\frac{x+\beta-\alpha-y}{x-y} \\
u=y+P, \quad v=x+P, \quad P=\frac{\beta-\alpha}{x-y}
\end{gathered}
$$

and

$$
\begin{array}{r}
u=y Q^{-1}, \quad v=x Q, \quad Q=\frac{(1-\beta) x y+(\beta-\alpha) y+\beta(\alpha-1)}{(1-\alpha) x y+(\alpha-\beta) x+\alpha(\beta-1)} \\
u=y Q^{-1}, \quad v=x Q, \quad Q=\frac{\alpha+(\beta-\alpha) y-\beta x y}{\beta+(\alpha-\beta) x-\alpha x y} \\
u=\frac{y}{\alpha} Q, \quad v=\frac{x}{\beta} Q, \quad Q=\frac{\alpha x+\beta y}{x+y} \\
u=y Q^{-1}, \quad v=x Q, \quad Q=\frac{\alpha x y+1}{\beta x y+1}
\end{array}
$$



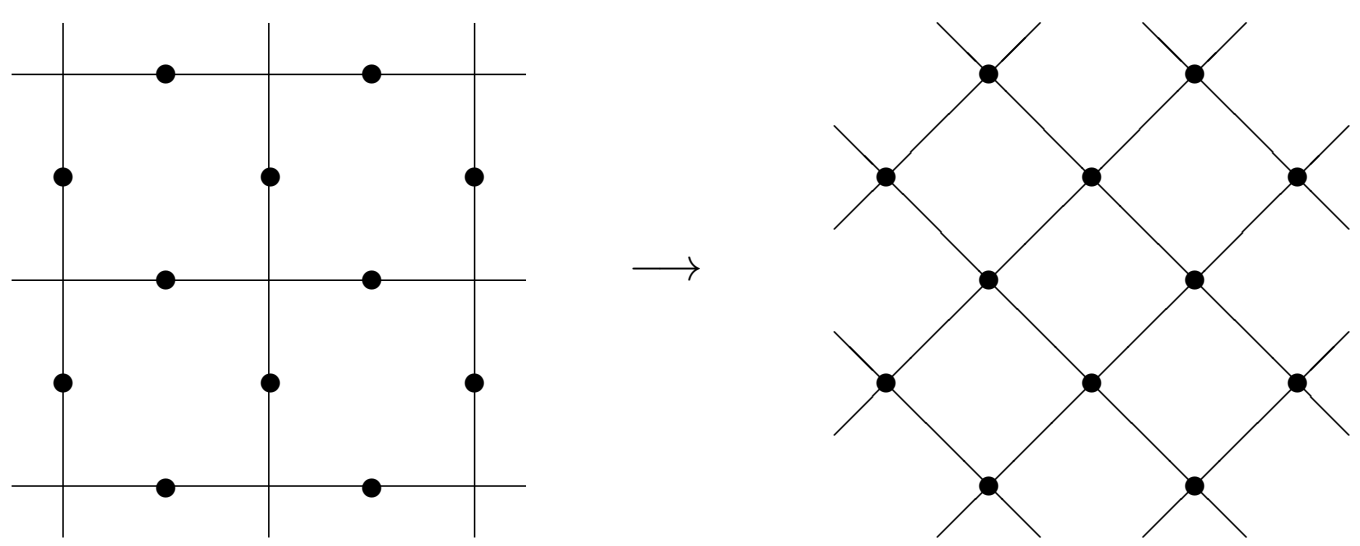

Fig. 2. A change of point of view

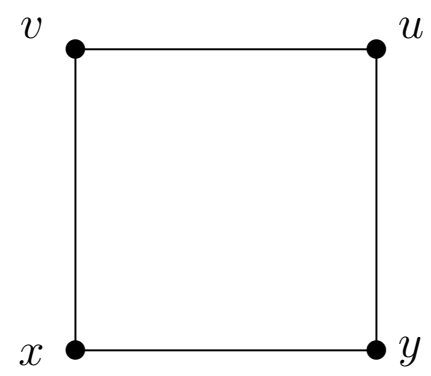

Fig. 3. New cell

$$
u=y-P, \quad v=x+P \quad P=\frac{\beta-\alpha}{x+y}
$$

We remark that the cases $H_{\mathrm{III}}^{\mathrm{A}}$ and $H_{\mathrm{V}}$ are equivalent respectively to $F_{\mathrm{III}}$ and $F_{\mathrm{V}}$, through the change $u \rightarrow-u$ and $v \rightarrow-v$.

\section{The median graph construction}

One may view the original lattice in a different way: the bond variables may be assigned to the vertices of a finer square lattice, as shown in Figure 3.

We get a new square lattice whose elementary square cell is, after rotation of $-\frac{\pi}{4}$ :

For all the models described in [9] the conditions relating $u, v, x, y$ allow to define various rational $2 \times 2$ maps: they give $u$ and $v$ in terms of $x$ and $y$, but also $x$ and $y$ in terms of $u$ and $v$, as well as $u$ and $y$ in terms of $v$ and $x$, or $v$ and $x$ in terms of $u$ and $y$. This is the "quadrirationality" property. We obtain in such a way four $2 \times 2$ maps, defined for each elementary cell. These cells form 


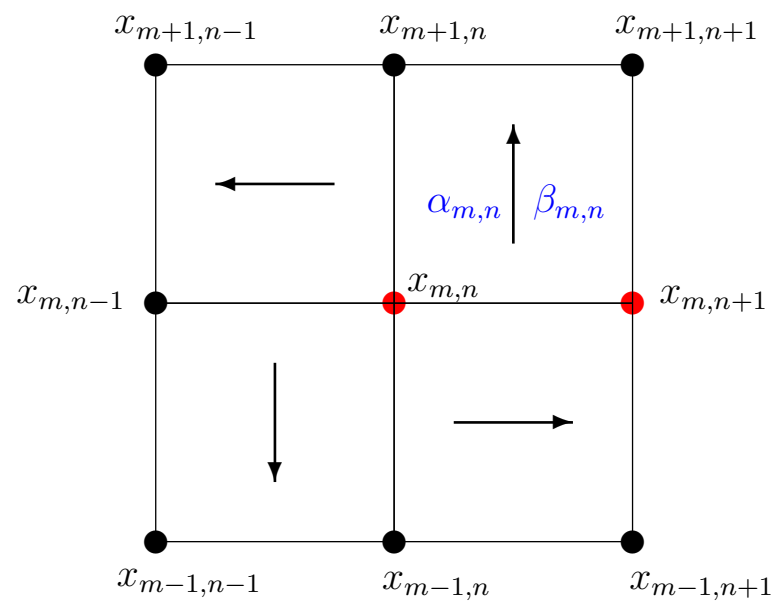

Fig. 4. Local compatibility condition around vertex $(m, n)$

a new two-dimensional lattice. Notice that there are two kinds of cells in this lattice. Half of them contained a vertex of the original lattice, and the other half did not. In the original lattice, we had relations only for the latter kind of cells. We will impose the existence of relations as above for all the cells, allowing non constant parameters. This is where the reduction takes place.

This will lead to a possibly non autonomous recurrence of order two. The variables, denoted by $x_{m, n}$, are assigned to the vertices of the new lattice. Since on each cell we have four $(m, n)$ dependent $2 \times 2$ maps, we may, once given initial conditions $x_{0,0}$ and $x_{0,1}$, calculate $x$ over the whole lattice, provided some compatibility (zero monodromy around each vertex) condition is fulfilled. The compatibility condition is a constraint on the (non-constant) parameters of the map, derived by requiring that compatibility be present for all initial values of the dependent variable.

We use the coordinates of the lower left corner to label the cells. Starting from $x_{m, n}$ and $x_{m, n+1}$, we compute $x_{m+1, n}$ and $x_{m+1, n+1}$ using the $F$ and $H$ equations given in the previous section, with $(m, n)$ dependent set of parameters $\Gamma_{m, n}$. Going around the vertex $(m, n)$ we calculate the surrounding values of $x$. This yields after four steps a value for $x_{m, n+1}$ which has to coincide with the initial condition.

Remark: Doing so we reduce the dimensionality of the system, going from partial difference equation to ordinary difference equation. The reason is that we impose relations between variables which were not related in the original model, actually exactly on half of the cells of the new lattice.

In what follows, we shall show that it is indeed possible to satisfy the compatibility constraint. Moreover the resulting equations are explicitly solvable ones and given that the compatibility constraints are also explicitly solvable this allows us to give the solution for all $x$ 's in closed form.

\section{Explicit solution of the diffference equations}

The dynamical interpretation of the $F$ and $H$ systems as median graphs is tantamount to that of defining a difference system with a parameter. Thus the two dimensional character would result from the evolution along what can be considered as the independent dynamical variable, on the one hand, and along contiguity relations between solutions for varying values of the parameter on the 
other. The image here resembles closely that of discrete Painlevé equations and their geometrical interpretation along what one considers the equation together with its Schlesinger transformations.

\section{The $F$ equations}

We start with $F_{\mathrm{V}}$ and the equations in the form

$$
u=y+P, \quad v=x+P, \quad P=\frac{\gamma}{x-y}
$$

where $\gamma=\alpha-\beta$, which we rewrite as

$$
x_{m+1, n+1}=x_{m, n+1}+\frac{\gamma_{m, n}}{x_{m, n}-x_{m, n+1}}, \quad x_{m+1, n}=x_{m, n}+\frac{\gamma_{m, n}}{x_{m, n}-x_{m, n+1}}
$$

The compatibility condition in this case turns out to be

$$
\gamma_{m+1, n+1} \gamma_{m, n}=\gamma_{m+1, n} \gamma_{m, n+1}
$$

the solution of which is $\gamma_{m, n}=\phi(m) \psi(n)$, where $\phi$ and $\psi$ are free functions of their argument. At this point is is convenient to introduce two functions $f(m)$ and $g(n)$ such that $f(m+1)-f(m)=$ $\phi(m)$ and $g(n+1)-g(n)=\psi(n)$. It is straightforward to verify that

$$
x_{m, n}=a f(m)+\frac{g(n)}{a}+b
$$

is the general solution of system (4.2), where $a, b$ are constants.

Next we turn to $F_{\mathrm{IV}}$

$$
u=y P, \quad v=x P, \quad P=1-\frac{\alpha-\beta}{x-y}
$$

which we rewrite as

$$
x_{m+1, n+1}=x_{m, n+1}\left(1-\frac{\gamma_{m, n}}{x_{m, n}-x_{m, n+1}}\right), \quad x_{m+1, n}=x_{m, n}\left(1-\frac{\gamma_{m, n}}{x_{m, n}-x_{m, n+1}}\right)
$$

with the same definition of $\gamma$ as before. The compatibility condition is exactly the same as in the previous case, namely (4.3). Here also we shall introduce the same auxiliary functions $f$ and $g$. The general solution here can be written as

$$
x_{m, n}=(f(m)+a)(g(n)+b)
$$

where $a, b$ are constants. 
In the case of $F_{\text {III }}$

$$
u=\frac{y}{\alpha} P, \quad v=\frac{x}{\beta} P, \quad P=\frac{\alpha x-\beta y}{x-y}
$$

we introduce the parameter $\gamma=\alpha / \beta$. We rewrite the equations as

$$
x_{m+1, n+1}=x_{m, n+1}\left(\frac{x_{m, n}-x_{m, n+1} / \gamma_{m, n}}{x_{m, n}-x_{m, n+1}}\right), \quad x_{m+1, n}=x_{m, n}\left(\frac{\gamma_{m, n} x_{m, n}-x_{m, n+1}}{x_{m, n}-x_{m, n+1}}\right)
$$

The compatibility condition is now

$$
\gamma_{m+1, n+1} \gamma_{m+1, n} \gamma_{m, n+1} \gamma_{m, n}-\gamma_{m+1, n} \gamma_{m, n+1} \gamma_{m, n}-\gamma_{m+1, n+1} \gamma_{m+1, n} \gamma_{m, n+1}+\gamma_{m+1, n}+\gamma_{m, n+1}-1=0
$$

which can be rewritten as

$$
\left(1-\gamma_{m+1, n+1}\right)\left(1-\gamma_{m, n}\right)=\left(1-1 / \gamma_{m+1, n}\right)\left(1-1 / \gamma_{m, n+1}\right)
$$

which can be recognized as the linearisable equation proposed by Hydon-Viallet, equation (22) of reference [8]. (The latter was originally given in terms of the variable $z=1 /(1-\gamma)$ ). The solution to (4.11) can be obtained most easily if we introduce an auxiliary variable $\omega$ and express $\gamma$ as

$$
\gamma_{m, n}=\frac{\omega_{m+1, n+1} \omega_{m, n}}{\omega_{m+1, n} \omega_{m, n+1}}
$$

and take $\omega$ satisfying the equation

$$
\omega_{m+1, n+1}+\omega_{m, n}=\omega_{m+1, n}+\omega_{m, n+1}
$$

i.e. $\omega$ is the sum of two free functions $\omega_{m, n}=f(m)+g(n)$ which leads to the solution for $\gamma$

$$
\gamma_{m, n}=\frac{(f(m+1)+g(n+1))(f(m)+g(n))}{(f(m+1)+g(n))(f(m)+g(n+1))}
$$

The general solution for $x$ can now be given

$$
x_{m, n}=a \frac{(f(m)+b)(g(n)-b)}{f(m)+g(n)}
$$

where $a, b$ are constants.

For the case $F_{\mathrm{II}}$ we start from the equations

$$
u=\frac{y}{\alpha} P, \quad v=\frac{x}{\beta} P, \quad P=\frac{\alpha x-\beta y+\beta-\alpha}{x-y}
$$

which we rewrite, introducing $\gamma=\alpha / \beta$, as

$$
\begin{aligned}
x_{m+1, n+1} & =x_{m, n+1}\left(\frac{x_{m, n}-x_{m, n+1} / \gamma_{m, n}+1-1 / \gamma_{m, n}}{x_{m, n}-x_{m, n+1}}\right), \\
x_{m+1, n} & =x_{m, n}\left(\frac{\gamma_{m, n} x_{m, n}-x_{m, n+1}+\gamma_{m, n}-1}{x_{m, n}-x_{m, n+1}}\right)
\end{aligned}
$$

The compatibility condition is exactly the same as before, namely $\gamma$ satisfies equation (4.11). The ansatz of $\gamma$ in terms of $\omega$ is the same but it is more convenient in this case to introduce another 
auxiliary variable $\chi$ by $\gamma_{m, n}=\frac{\left(1-\chi_{m+1, n+1}\right)\left(1-\chi_{m, n}\right)}{\left(1-\chi_{m+1, n}\right)\left(1-\chi_{m, n+1}\right)}$, with $\chi$ satisfying the equation

$$
\chi_{m+1, n+1} \chi_{m, n}=\chi_{m+1, n} \chi_{m, n+1}
$$

i.e. $\chi$ is the product of two free functions $\chi_{m, n}=f(m) g(n)$. Using this solution we can obtain the general solution for $x$ as

$$
x_{m, n}=\frac{a f(m) g(n)+b f(m)+c g(n)+a+1}{1-f(m) g(n)}
$$

where $a, b, c$ are constants related by $a(a+1)=b c$.

In the case of $F_{\mathrm{I}}$ the two parameters $\alpha$ and $\beta$ cannot be combined into a single parameter

$$
u=\alpha y P, \quad v=\beta x P, \quad P=\frac{(1-\beta) x+\beta-\alpha+(\alpha-1) y}{\beta(1-\alpha) x+(\alpha-\beta) x y+\alpha(\beta-1)}
$$

which we rewrite as

$$
\begin{gathered}
x_{m+1, n+1}=\alpha_{m, n} x_{m, n+1} \frac{\left(1-\beta_{m, n}\right) x_{m, n}+\beta_{m, n}-\alpha_{m, n}+\left(\alpha_{m, n}-1\right) x_{m, n+1}}{\beta_{m, n}\left(1-\alpha_{m, n}\right) x_{m, n}+\left(\alpha_{m, n}-\beta_{m, n}\right) x_{m, n} x_{m, n+1}+\alpha_{m, n}\left(\beta_{m, n}-1\right) x_{m, n+1}} \\
x_{m+1, n}=\beta_{m, n} x_{m, n} \frac{\left(1-\beta_{m, n}\right) x_{m, n}+\beta_{m, n}-\alpha_{m, n}+\left(\alpha_{m, n}-1\right) x_{m, n+1}}{\beta_{m, n}\left(1-\alpha_{m, n}\right) x_{m, n}+\left(\alpha_{m, n}-\beta_{m, n}\right) x_{m, n} x_{m, n+1}+\alpha_{m, n}\left(\beta_{m, n}-1\right) x_{m, n+1}}
\end{gathered}
$$

The compatibility conditions for the $\alpha$ and $\beta$ are

$$
\alpha_{m+1, n+1}=\alpha_{m, n}, \quad \beta_{m+1, n}=\beta_{m, n+1}
$$

and

$$
\alpha_{m+1, n}=\beta_{m, n} \beta_{m, n+1} R, \quad \beta_{m+1, n+1}=\alpha_{m, n} \alpha_{m, n+1} R
$$

where

$$
R=\frac{\left(\alpha_{m, n}-1\right)\left(\alpha_{m, n+1}-1\right)-\left(\beta_{m, n}-1\right)\left(\beta_{m, n+1}-1\right)}{\alpha_{m, n} \alpha_{m, n+1}\left(\beta_{m, n}+\beta_{m, n+1}-1\right)-\beta_{m, n} \beta_{m, n+1}\left(\alpha_{m, n}+\alpha_{m, n+1}-1\right)}
$$

The set of compatibility equations is itself overdetermined but it turns out that it is consistent and the solution is periodic in both $m$ and $n$. We have

$$
\begin{aligned}
& \alpha_{3 m, 3 n}=\alpha_{3 m+1,3 n+1}=\alpha_{3 m+2,3 n+2}=\alpha_{0} \\
& \alpha_{3 m, 3 n+1}=\alpha_{3 m+1,3 n+2}=\alpha_{3 m+2,3 n}=\alpha_{1} \\
& \alpha_{3 m, 3 n+2}=\alpha_{3 m+1,3 n}=\alpha_{3 m+2,3 n+1}=\alpha_{2} \\
& \beta_{3 m, 3 n}=\beta_{3 m+2,3 n+1}=\beta_{3 m+1,3 n+2}=\beta_{0} \\
& \beta_{3 m, 3 n+1}=\beta_{3 m+2,3 n+2}=\beta_{3 m+1,3 n}=\beta_{1} \\
& \beta_{3 m, 3 n+2}=\beta_{3 m+2,3 n}=\beta_{3 m+1,3 n+1}=\beta_{2}
\end{aligned}
$$

where $\alpha_{2}, \beta_{2}$ are given in terms of $\alpha_{0}, \alpha_{1}, \beta_{0}, \beta_{1}$ using (4.24). The solution for $x_{m, n}$ is equally periodic, with period 3 in both $m$ and $n$ directions however in this case all nine $x_{3 m+j, 3 m+k}$ for $j, k=0,1,2$ are generically different. 


\section{The $H$ equations}

We can now turn to the remaining equations of the $H$ family and start with $H_{\mathrm{V}}$

$$
u=y-P, \quad v=x+P, \quad P=\frac{\gamma}{x+y}
$$

where $\gamma=\alpha-\beta$, which we rewrite as

$$
x_{m+1, n+1}=x_{m, n+1}-\frac{\gamma_{m, n}}{x_{m, n}+x_{m, n+1}}, \quad x_{m+1, n}=x_{m, n}+\frac{\gamma_{m, n}}{x_{m, n}+x_{m, n+1}}
$$

Next we introduce a new variable $X_{m, n}=(-1)^{n} x_{m, n}$ whereupon (4.28) becomes identical to (4.2), written for $X$. Thus the solution of (4.28) is given by (4.4) with an overall factor $(-1)^{n}$.

The same change of variables allows us to relate $H_{\mathrm{III}}^{\mathrm{A}}$

$$
u=\frac{y}{\alpha} Q, \quad v=\frac{x}{\beta} Q, \quad Q=\frac{\alpha x+\beta y}{x+y}
$$

or equivalently

$$
x_{m+1, n+1}=x_{m, n+1}\left(\frac{x_{m, n}+x_{m, n+1} / \gamma_{m, n}}{x_{m, n}+x_{m, n+1}}\right), \quad x_{m+1, n}=x_{m, n}\left(\frac{\gamma_{m, n} x_{m, n}+x_{m, n+1}}{x_{m, n}+x_{m, n+1}}\right)
$$

to $F_{\mathrm{III}}$, equation (4.9), and thus to obtain its solution from (4.15) again with an overall factor $(-1)^{n}$.

Equation $H_{\mathrm{III}}^{\mathrm{B}}$

$$
u=\frac{y}{Q}, \quad v=x Q, \quad Q=\frac{\alpha x y+1}{\beta x y+1}
$$

is special in the sense that the compatibility condition must be obtained on $\alpha$ and $\beta$ separately. We start from

$$
x_{m+1, n+1}=x_{m, n+1} \frac{\beta_{m, n} x_{m, n} x_{m, n+1}+1}{\alpha_{m, n} x_{m, n} x_{m, n+1}+1}, \quad x_{m+1, n}=x_{m, n} \frac{\alpha_{m, n} x_{m, n} x_{m, n+1}+1}{\beta_{m, n} x_{m, n} x_{m, n+1}+1}
$$

and obtain the compatibility conditions. The latter can be written in more compact form if we introduce $\alpha=\gamma+\delta$ and $\beta=\gamma-\delta$. We obtain

$$
\begin{gathered}
\delta_{m, n} \delta_{m+1, n+1}=\delta_{m, n+1} \delta_{m+1, n} \\
\left(\gamma_{m+1, n}-\gamma_{m, n}\right) \delta_{m+1, n+1}+\left(\gamma_{m+1, n+1}-\gamma_{m, n+1}\right) \delta_{m+1, n}=0
\end{gathered}
$$

which can be readily integrated to

$$
\begin{aligned}
& \alpha_{m, n}=f(n)\left(h(n)+(-1)^{n} k(m)+g(m)\right) \\
& \beta_{m, n}=f(n)\left(h(n)+(-1)^{n} k(m)-g(m)\right)
\end{aligned}
$$

Next we remark that from (4.32) we have

$$
x_{m, n} x_{m, n+1}=x_{m+1, n} x_{m+1, n+1} \equiv \phi(n)
$$

where $\phi$ is, obviously, independent of $m$. Given the expressions of $\alpha$ and $\beta$ we can obtain the value of $1 / \phi=f(n)\left(-h(n)+(-1)^{n} c\right)$, where $c$ is an integration constant, the value of which is fixed by 
the initial conditions. The values of $x$ can now be obtained from the recursion

$$
x_{m, n+2}=\frac{\phi(n+1)}{\phi(n)} x_{m, n}
$$

Using the expression for $\phi$ we can, starting from some initial condition $x_{0,0}, x_{0,1}$ obtain all $x_{0, n}$ for all $n$. Once the $x_{0, n}$ are obtained we can compute $x_{m, n}$ by

$$
\begin{gathered}
x_{m+1,2 n}=\frac{c-k(m)-g(m)}{c-k(m)+g(m)} x_{m, 2 n} \\
x_{m+1,2 n+1}=\frac{c-k(m)+g(m)}{c-k(m)-g(m)} x_{m, 2 n+1}
\end{gathered}
$$

Thus the solution to (4.32) can be expressed as a double infinite product.

The last but one case we shall examine is that of $H_{\mathrm{II}}$

$$
u=y Q^{-1}, \quad v=x Q, \quad Q=\frac{\alpha+(\beta-\alpha) y-\beta x y}{\beta+(\alpha-\beta) x-\alpha x y}
$$

which can be cast into the form

$$
\begin{aligned}
x_{m+1, n+1} & =x_{m, n+1} \frac{1+\left(\gamma_{m, n}-1\right) x_{m, n}-\gamma_{m, n} x_{m, n} x_{m, n+1}}{\gamma_{m, n}+\left(1-\gamma_{m, n}\right) x_{m, n+1}-x_{m, n} x_{m, n+1}} \\
x_{m+1, n} & =x_{m, n} \frac{\gamma_{m, n}+\left(1-\gamma_{m, n}\right) x_{m, n+1}-x_{m, n} x_{m, n+1}}{1+\left(\gamma_{m, n}-1\right) x_{m, n}-\gamma_{m, n} x_{m, n} x_{m, n+1}}
\end{aligned}
$$

where $\gamma=\alpha / \beta$. It turns out that the compatibility condition for $\gamma$ is precisely (4.10) i.e. the HydonViallet equation. We give the solution for $\gamma$ in the same form as in the case of $F_{\text {II }}$, namely

$$
\gamma_{m, n}=\frac{(1-f(n+1) g(m+1))(1-f(n) g(m))}{(1-f(n+1) g(m))(1-f(n) g(m+1))}
$$

We can now give the solution for $x$ which turns out to possess an even-odd freedom

$$
x_{m, n}=\frac{\left(1+a_{n} f(n)\right)\left(g(m)+a_{n+1}\right)}{\left(1+a_{n+1} f(n)\right)\left(g(m)+a_{n}\right)}
$$

where $a_{n}$ is a period-two constant, i.e. $a_{n+2}=a_{n}$.

The last case we are going to examine is $H_{\mathrm{I}}$

$$
u=y Q^{-1}, \quad v=x Q, \quad Q=\frac{(1-\beta) x y+(\beta-\alpha) y+\beta(\alpha-1)}{(1-\alpha) x y+(\alpha-\beta) x+\alpha(\beta-1)}
$$

which we rewrite, after a translation $\alpha=\gamma+1, \beta=\delta+1$, as

$$
\begin{aligned}
x_{m+1, n+1} & =x_{m, n+1} \frac{\gamma_{m, n} x_{m, n} x_{m, n+1}-\left(\gamma_{m, n}-\delta_{m, n}\right) x_{m, n}-\delta_{m, n}\left(\gamma_{m, n}+1\right)}{\delta_{m, n} x_{m, n} x_{m, n+1}+\left(\gamma_{m, n}-\delta_{m, n}\right) x_{m, n+1}-\gamma_{m, n}\left(\delta_{m, n}+1\right)} \\
x_{m+1, n} & =x_{m, n} \frac{\delta_{m, n} x_{m, n} x_{m, n+1}+\left(\gamma_{m, n}-\delta_{m, n}\right) x_{m, n+1}-\gamma_{m, n}\left(\delta_{m, n}+1\right)}{\gamma_{m, n} x_{m, n} x_{m, n+1}-\left(\gamma_{m, n}-\delta_{m, n}\right) x_{m, n}-\delta_{m, n}\left(\gamma_{m, n}+1\right)}
\end{aligned}
$$


In this case the two parameters $\gamma$ and $\delta$ cannot be combined into a single parameter. The compatibility condition turns out to be

$$
\gamma_{m+1, n}=\delta_{m, n}, \quad \delta_{m+1, n}=\gamma_{m, n}
$$

which means that $\gamma$ and $\delta$ can be expressed in terms of two free functions $f, g$ of $n$ alone: $\gamma_{2 m, n}=$ $\delta_{2 m+1, n}=f(n), \gamma_{2 m+1, n}=\delta_{2 m, n}=g(n)$. The solution of (4.47), (4.48) is also periodic in the $m$ direction: $x_{m+2, n}=x_{m, n}$. Thus the equation becomes a one-dimensional one for the variable $x$ at given parity of $m$. Once we have obtained the solution for one parity the solution at the other parity is obtained through (4.47) or (4.48). Taking the index $m$ to be even we obtain the equation for $x$

$$
\begin{gathered}
(g(n) g(n-1)-f(n) f(n-1)) x_{n-1} x_{n} x_{n+1}+g(n)(f(n-1)-g(n-1)) x_{n} x_{n+1} \\
+f(n-1)(f(n)-g(n)) x_{n} x_{n-1}+f(n-1)(g(n-1)+1)(f(n)-g(n)) x_{n+1} \\
+g(n)(f(n)+1)(f(n-1)-g(n-1)) x_{n-1}+g(n) g(n-1)(f(n)+f(n-1)+1) \\
-f(n) f(n-1)(g(n)+g(n-1)+1)=0
\end{gathered}
$$

where we have dropped the even index $m$. (In fact the equation for odd- $m$ is the same up to the permutation of $f$ and $g$ ). Equation (4.50) is linearisable. A study of the growth of the degrees of the iterates on an initial condition, using algebraic entropy techniques, leads to the following sequence of degrees: $0,1,1,2,2,3,3,4,4,5, \ldots$. This suggest that the mapping (4.50) is of Gambier type [10] and this turns out to be indeed the case. We find for (4.50) the invariant

$$
K=(-1)^{n} \frac{f(n)\left(x_{n}+1\right)\left(x_{n+1}-1\right)+g(n)\left(x_{n}-1\right)\left(x_{n+1}+1\right)-2 f(n) g(n)}{(f(n)-g(n))\left(x_{n}-1\right)\left(x_{n+1}-1\right)}
$$

which allows to reduce (4.50) to a homographic mapping.

This completes the integration of the equations of the $F$ and $H$ families in their interpretation as median graph reductions.

\section{Conclusion}

Integrable discrete equations appear in an innumerable number of avatars. It is no surprise that the Yang-Baxter maps possess various dynamical interpretations. In this paper we have addressed this question and proposed a dynamical interpretation which reduces the Yang-Baxter maps to ordinary difference equations. It turned out that in all cases the equations obtained were linearisable, provided some compatibility condition was satisfied by the parameters of the equation. An interesting result was the appearance, in the compatibility condition, of a linearisable lattice equation proposed in [8] and integrated in [7]. In all cases we have been able to present the general solution of the system.

The study presented here cannot be exhaustive. There are other types of transformations establishing relations between different integrable systems, and the links between these various correspondences and the one we presented here still need to be explored.

\section{Acknowledgment}

We would like to thank Jarmo Hietarinta for stimulating discussions in the early stage of this work.

\section{References}

[1] H. Sakai, Rational Surfaces Associated with Affine Root Systems and Geometry of the Painlevé Equations, Comm. Math. Phys. 220(1) (2001), pp. 165-229. 
[2] G.R.W. Quispel, J.A.G. Roberts and C.J. Thompson, Integrable Mappings and Soliton Equations II, Physica D34 (1989), pp. 183-192.

[3] A. Ramani, B. Grammaticos and J. Hietarinta, Discrete versions of the Painlevé Equations, Phys. Rev. Lett. 67 (1991), pp. 1829-1832.

[4] V.E. Adler, A.I. Bobenko and Yu.B. Suris, Classification of integrable equations on quad-graphs. The consistency approach, Comm. Math. Phys. 233(3) (2003), pp. 513-543.

[5] V.E. Adler, A.I. Bobenko and Yu.B. Suris, Discrete nonlinear hyperbolic equations. Classification of integrable cases, Funct. Anal. Appl. 43 (2009), pp. 3-17.

[6] V.G. Papageorgiou, Y.B. Suris, A.G. Tongas and A.P. Veselov, On quadrirational Yang-Baxter maps, Sigma 6 (2010), 033 (9 pp).

[7] C. Scimiterna, A. Ramani and B. Grammaticos, On two integrable lattice equations and their interpretation, J. Phys. A FTC 44 (2011), 032002 (6 pp).

[8] P.E. Hydon and C.-M. Viallet, Asymmetric integrable quad-graph equations, Applicable Analysis 89(4) (2010), pp. 493-506.

[9] V.E. Adler, A.I. Bobenko and Yu.B. Suris, Geometry of Yang-Baxter maps: pencils of conics and quadrirational mappings, Commun. Anal. Geom. 12 (2004), pp. 967-1007.

[10] B. Grammaticos and A. Ramani, The Gambier Mapping, Physica A 223 (1995), p. 125-136. 